\title{
Clinical efficacy of propranolol in the treatment of hemangioma and changes in serum VEGF, bFGF and MMP-9
}

\author{
SHANYING WU, BIAO WANG, LIFEN CHEN, SHUYUAN XIONG, FULIAN ZHUANG, \\ XUNLEI HUANG, MEISHUI WANG and ZUGEN HUANG
}

Department of Plastic Surgery, The First Affiliated Hospital of Fujian Medical University, Fuzhou, Fujian 350001, P.R. China

Received September 1, 2014; Accepted June 12, 2015

DOI: $10.3892 /$ etm.2015.2650

\begin{abstract}
The aim of this study was to observe the clinical efficacy and safety of propranolol in the treatment of hemangioma, and to reveal its possible mechanism. A total of 129 cases of proliferative hemangioma were divided into two groups: i) Treatment $(n=97)$, in which the patients received oral propranolol therapy and ii) observation $(n=32)$, in which the patients underwent clinical observation. The changes in the hemangiomas were noted and compared between the two groups. In addition, the heart rate, blood glucose levels, liver, kidney and thyroid function of the patients in the treatment group were monitored prior to and following treatment; the ELISA method was used for the measurement of the patients' serum concentrations of vascular endothelial growth factor (VEGF), basic fibroblast growth factor (bFGF) and matrix metalloproteinase-9 (MMP-9) at the same time-points. A significant difference in curative effect was found between the treatment and observation group. The concentration of free thyroxine and sensitive thyroid-stimulating hormone and the heart rate of the treatment group exhibited significant changes prior to and following medication, but no statistical significance was found in the changes in blood glucose, liver and kidney function and free triiodothyronine concentration. Furthermore, the serum concentrations of VEGF, bFGF and MMP-9 in the treatment group 8 weeks after medication were decreased significantly compared with those before treatment. In conclusion, oral propranolol has a good curative effect in the treatment of proliferative hemangioma, with few side effects and a high level of safety. The mechanism underlying the effects of propranolol may be associated with the downregulation of VEGF, bFGF and MMP-9 expression.
\end{abstract}

Correspondence to: Dr Biao Wang, Department of Plastic Surgery, The First Affiliated Hospital of Fujian Medical University, 20 Chazong Road, Fuzhou, Fujian 350001, P.R. China

E-mail: biaowangdoc@163.com

Key words: propranolol, hemangioma, infant, vascular endothelial growth factor, basic fibroblast growth factor, matrix metalloproteinase-9

\section{Introduction}

Hemangioma is one of the most common types of infantile benign tumor and is a congenital dysplasia that appears in the process of embryonic blood vessel formation. The incidence rate of all newborns is $1.1-2.6 \%$ (1), and the condition, which usually occurs in the head and neck of infants, is more common in premature infants, particularly females. According to the biological characteristics of the endothelial cells in hemangioma and its clinical manifestations, the natural course of the condition can be divided into the proliferating, involuting and involuted phases $(2,3)$. Hemangiomas at the proliferative stage exhibit rapid growth and may affect the normal development of the body. The development of hemangioma in certain parts of the body may affect the appearance of the patient, have an impact on the normal function of body and even prove life-threatening; therefore, such hemangiomas require aggressive treatment promptly $(4,5)$.

At present, with the improvement in living standards, noninvasive methods of treatment tend to be preferred by patients; therefore, drug treatments are commonly selected. Drug therapy includes topical drug therapy and oral medication. In 2008, Léauté-Labrèze et al (6) found that the $\beta$-receptor blocker propranolol was able to treat infantile hemangioma; with the exception of individual cases of low blood pressure, no serious adverse reactions were observed in the other children throughout the treatment course. Furthermore, clinical practice in recent years has shown that propranolol exerts a rapid, curative effect in the treatment of hemangioma, with few inter-patient differences and adverse reactions; however, the underlying therapeutic mechanism is not clear. The aim of the present study, therefore, was to assess the clinical efficacy and safety of propranolol in the treatment of infantile hemangioma by monitoring clinical and biochemical parameters. Furthermore, the study aimed to investigate the mechanism underlying the effects of propranolol by measuring the serum expression levels of vascular endothelial growth factor (VEGF), basic fibroblast growth factor (bFGF) and matrix metalloproteinase-9 (MMP-9) prior to and following medication.

\section{Patients and methods}

Patient inclusion. Between September 2009 and December 2012, 129 patients with newly diagnosed cases of infantile 
hemangioma (both in- and outpatients) were enrolled as the study subjects. For inclusion in the study, the patients had to be aged $<1$ year and exhibit the following characteristics of hemangioma onset: i) Mosquito bite- or pin-like red spots at birth or 7-10 days later or the appearance of patchy erythema at birth, with patchy expansion or subcutaneous soft masses; ii) a rapid tumor growth history; and iii) a strawberry or light blue tumor surface that fades or reduces in color when pressed. Patients with certain diseases, including sinus bradycardia, bronchial asthma, cardiogenic shock, heart block (type II-III atrioventricular block) and severe or acute heart failure, were excluded from the study.

Grouping. The infants were assigned to either the treatment group or the observation group according to the approval of the family of each patient. The observation group comprised 32 cases, including 11 male patients and 21 female patients. The patients were aged between 1 month, 10 days and 10 months, with an average age of 105 days. The location of the hemangiomas was as follows: Head and neck, 13 cases; limbs, 8 cases; trunk, 8 cases; and perineum, 3 cases. The treatment group comprised 97 cases, including 31 male patients and 66 female patients, with ages ranging between 24 days and 11 months, 2 days (average, 105 days). The patients' weights ranged between 3.8 and $10.0 \mathrm{~kg}$, with an average body weight of $6.07 \mathrm{~kg}$. The weight for observation group ranged between 3.8 and $9.2 \mathrm{~kg}$, with an average body weight of $6.30 \mathrm{~kg}$. The location of the hemangiomas was as follows: Head and neck, 59 cases; limbs, 14 cases; trunk, 12 cases; perineum, 5 cases; and multiple sites, 7 cases.

Data collection. The patients of the observation group were clinically followed-up and generally reviewed once every 2-4 weeks, in order to measure the size of the tumor and observe the changes in color and tension.

The patients of the treatment group were admitted and the family of each patient was informed of the objectives of the oral propranolol treatment, as well as the safety and associated risks. Written consent was obtained prior to treatment. Once the relevant examinations had been performed to confirm that no contraindications to propranolol treatment existed, the treatment was started at the dose of $1.5 \mathrm{mg} / \mathrm{kg}$ per day (Shijiazhuang Pharmaceutical Group Co., Ltd., Hebei, China). The propranolol was administered once a day in the form of a draught in the morning. During the treatment period, the heart rate of each patient was measured prior to and 1, 3 and $6 \mathrm{~h}$ after medication for a total of 3 days. In addition, the blood glucose levels prior to and $2 \mathrm{~h}$ after medication were monitored, again for a total of 3 days. If the infants had exhibited no obvious abnormal reaction after 3 days, they were discharged from hospital to continue the medication until tumor regression was observed. During this period, the liver and renal function and the thyroid function were assessed 4 and 8 weeks after the start of medication, respectively, the tumor size was measured and changes in the color and tension of the hemangioma were observed. Any changes in the mental status, appetite, stool appearance or weight of the patients during medication were additionally noted during the follow-up.

Sample collection and testing. Following the provision of informed consent by the family members, the blood samples
( $2 \mathrm{ml}$, without anticoagulant) of 40 infants in the treatment group were collected prior to and 8 weeks after medication. The samples were placed in the test tube, kept static at room temperature for $30 \mathrm{~min}$ and then subjected to centrifugation at $658.44 \mathrm{x} \mathrm{g}$ to isolate the serum. The serum was placed into an Eppendorf (EP) tube and preserved at $-80^{\circ} \mathrm{C}$ for subsequent testing. Following the collection of the samples, the serum concentrations of VEGF, bFGF and MMP-9 were detected using the ELISA method.

Experimental reagents and consumables. The standard equipment used in the study included a centrifuge, balance, EP tubes, freezer $\left(-80^{\circ} \mathrm{C}\right)$, enzyme mark instrument $(450 \mathrm{~nm})$, high-precision pipette and gun head, constant temperature box $\left(37^{\circ} \mathrm{C}\right)$, disposable tubes and absorbent paper. The human VEGF, bFGF and MMP-9 ELISA kits were obtained from Beijing Dingguochangsheng Biotechnology Co., Ltd. (Beijing, China).

Clinical efficacy criteria. The grading criteria proposed by Achauer et al (7) were used to analyze the effect of the treatment/observation on the hemangiomas. The results were obtained after 8 weeks of treatment or observation. The four grades were as follows: Grade I (poor), reduction in tumor size of $<25 \%$; Grade II (medium), reduction in tumor size and skin lesion color of 26-50\%; Grade III (good), reduction in tumor size and skin lesion color of 51-75\%; Grade IV (excellent), reduction in tumor size and skin lesion color of $>75 \%$ (disappearance of the tumor and restoration or near-restoration of the skin lesion color to that of normal skin). Grades II-IV (medium, good and excellent) were considered to represent an effective response, while Grade I (poor) was an invalid response.

Statistical analysis. The data were analyzed using SPSS 17.0 statistical software (SPSS, Inc., Chicago, IL, USA). Count data were compared using the $\chi^{2}$ test, and measurement data were compared using a paired-samples t-test. $\mathrm{P}<0.05$ was considered to indicate a statistically significant difference.

\section{Results}

Clinical efficacy observation. In the treatment group, the longest period of propranolol administration was 1 year; however, for the convenience of the study and statistical analysis, the results were taken after 8 weeks of medication. Following treatment or observation for 8 weeks, the results showed the curative effect of the treatment group to be excellent in 27 cases (Fig. 1), good in 34 cases, medium in 35 cases and poor in 1 case, with a total efficiency of $98.97 \%$. By comparison, the curative effect of the observation group was excellent in 0 cases, good in 0 cases, medium in 10 cases and poor in 22 cases, with the total efficiency of $31.25 \%$. The efficacy of the treatment group was significantly higher than that of the observation group $(\mathrm{P}<0.05)$ (Table I).

Changes in the serum concentrations of VEGF, bFGF and $M M P-9$ prior to and following treatment. The serum concentrations of VEGF, bFGF and MMP-9 in the children after 8 weeks of therapy were decreased significantly compared 
Table I. Comparison of the curative effects between the treatment group and the observation group.

\begin{tabular}{lcccccc}
\hline Group & Total cases (n) & Excellent (n) & Good (n) & Medium (n) & Poor (n) & Total efficacy (\%) \\
\hline Treatment & 97 & 27 & 34 & 35 & 1 & $98.97^{\mathrm{a}}$ \\
Observation & 32 & 0 & 0 & 10 & 22 & 31.25 \\
\hline
\end{tabular}

${ }^{\mathrm{a}} \mathrm{P}<0.05$ vs. the observation group.

Table II. Changes in the serum concentrations of VEGF, bFGF and MMP-9 in the treatment group before and 8 weeks after medication.

\begin{tabular}{lcc}
\hline Protein & Before medication & 8 weeks after medication \\
\hline VEGF $(\mathrm{pg} / \mathrm{ml})$ & $264.1825 \pm 15.32993$ & $142.6950 \pm 18.83824^{\mathrm{a}}$ \\
bFGF $(\mathrm{pg} / \mathrm{ml})$ & $59.4100 \pm 4.02030$ & $38.8450 \pm 5.83790^{\mathrm{a}}$ \\
MMP-9 $(\mathrm{ng} / \mathrm{ml})$ & $411.2080 \pm 28.35919$ & $279.9350 \pm 29.79854^{\mathrm{a}}$ \\
\hline
\end{tabular}

${ }^{\text {a }}<0.05$ vs. before medication. VEGF, vascular endothelial growth factor; bFGF, basic fibroblast growth factor; MMP-9, matrix metalloproteinase-9.
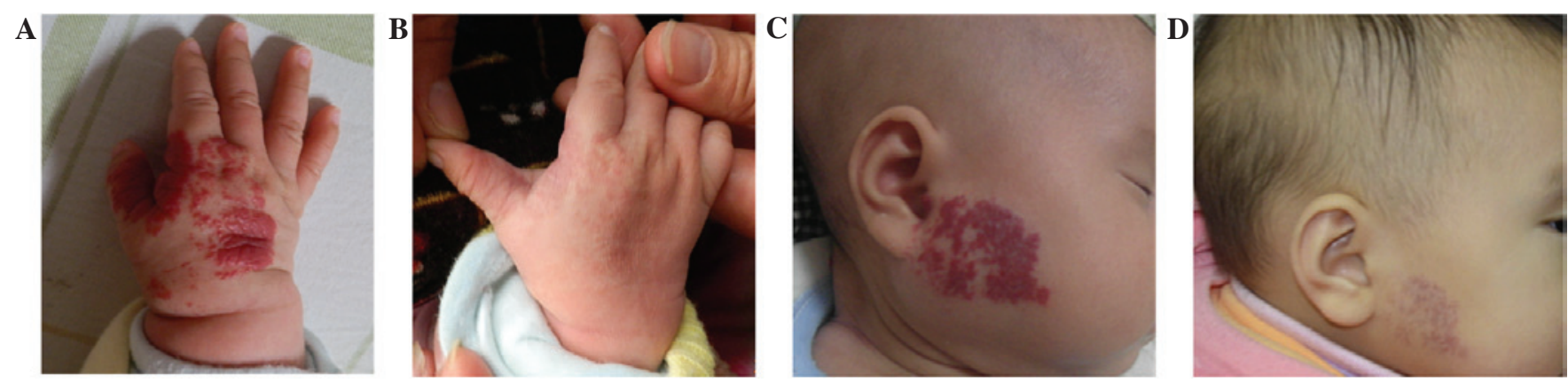

Figure 1. Changes in hemangiomas before and 8 weeks after propranolol treatment. (A and C) Before medication; (B and D) 8 weeks after medication.

with those prior to treatment. The concentration changes were statistically significant $(\mathrm{P}<0.05)$ (Table II).

Changes in heart rate and biochemical parameters prior to and following treatment. During the 3 days of hospitalization, the heart rates of the patients in the treatment group before and 1, 3 and $6 \mathrm{~h}$ after medication exhibited significant differences; however, the clinical observation revealed no bradycardia or other symptoms. In addition, the blood glucose levels and liver and renal function prior to and following medication exhibited no significant changes. With regard to the thyroid function, the comparison of the free triiodothyronine (FT3) levels prior to and following treatment did not show a significant difference, but the changes in the concentrations of free thyroxine (FT4) and sensitive thyroid-stimulating hormone (sTSH) did have statistical significance: The serum concentration of FT4 following medication was higher than that prior to medication, but mostly remained within the normal range, and the serum concentration of STSH following medication was lower than that prior to medication, but again mostly remained within the normal range. No clinical symptoms developed during the medication period.
Side effects. During medication, infants of the treatment groups showed good spirits. Seven patients exhibited a slight loss of appetite, diarrhea and other symptoms, which required no special treatment and were self-healing, and no other adverse reactions were observed.

\section{Discussion}

Hemangiomas are a type of common, benign tumor associated with the abnormal proliferation of vascular endothelial cells. Hemangiomas are caused by uncontrolled vascular generation or an imbalance of angiogenic cytokines and angiogenesis inhibitors. VEGF, bFGF and MMP-9 play an important role in the formation of hemangiomas (4,8-10): VEGF is a type of glycosylated secreted polypeptide factor that can induce endothelial cell proliferation and migration, enhance the permeability of blood vessels and promote the growth of new vessels and stromal cells $(8,11)$; bFGF is a type of strong endothelial cell and wall connective tissue growth factor, which can promote the migration of endothelial cells into the extracellular matrix to form capillary tissues and induce the secretion of VEGF (11); MMPs are a class of zinc and calcium-dependent 
extracellular proteolytic enzymes, which not only can promote the release of the angiogenic factors VEGF and bFGF to induce angiogenesis, but also can degrade the extracellular matrix and promote the migration of endothelial cells and the formation of the tube structure (4).

The association between serum VEGF concentration and endothelial cell proliferation in hemangioma has been demonstrated previously $(8,9)$, with the serum VEGF concentration of proliferative hemangiomas found to be significantly higher than that of involuting hemangiomas, vascular malformation and the normal control group. In the present study, the serum concentrations of VEGF, bFGF and MMP-9 8 weeks after medication were significantly reduced compared with those prior to treatment, and the results of this study were consistent with those of previous studies $(4,10)$. The reduction in the concentration of these three proteins is associated with the termination or regression of the growth of the hemangioma, which indicates that one of the mechanisms of propranolol in the treatment of proliferative hemangiomas may involve the downregulation of VEGF, bFGF and MMP-9; however, the exact mechanism remains to be elucidated. It has previously been found that VEGF expression is regulated by the extracellular signal-regulated kinase (ERK)/mitogen-activated protein kinase (MAPK) pathway (12), and protein kinase A (PKA) is a type of cyclic adenosine-3',5'-monophosphate (cAMP)-dependent protein kinase (13) that can promote VEGF secretion through the activation of the ERK/MAPK pathway. cAMP is the product of ATP following the action of cell adenylate cyclase, which is the earliest determined intracellular second messenger. cAMP is involved in a variety of the metabolic processes of the body enzymes, which can not only regulate the division, differentiation and development of cells, but also can regulate the expression of genes and promote the transcription of mRNA (14). cAMP can activate cAMP-dependent PKA, and PKA enables intracellular protein phosphorylation, regulating the activity of the proteins and promoting their reactions; thus, a model of a cAMP/PKA signal transduction pathway is formed. A previous study (5) suggested that, through the inhibition of the cAMP/PKA signaling pathway on the activation of the ERK/MAPK signaling pathway, propranolol could inhibit the secretion of VEGF in proliferative hemangioma. In a different study (15), it was suggested that, by downregulating the expression of the Raf/MAPK pathway, propranolol could decrease the expression of VEGF and bFGF in the late period of hemangioma treatment, so as to promote the further regression of the hemangioma; however, further study is required to confirm these theories.

The results of the present study revealed a significant difference between the curative effect of the treatment group and that of the observation group, which confirmed the validity of propranolol in the treatment of proliferative hemangioma. The comparison of the heart rate of 97 infants with hemangioma prior to propranolol treatment and 1,3 and $6 \mathrm{~h}$ after medication also revealed statistically significant differences; however, no bradycardia or other symptoms were observed. No significant changes were found between the blood glucose levels, and liver and renal function prior to and following medication; with regard to the thyroid function, the concentration of FT3 exhibited no significant changes prior to and following treatment, but the changes in the concentrations of FT4 and STSH were significant. We consider that oral propranolol does not affect the secretion of thyroid hormones, but can inhibit the conversion of T4 into T3 in peripheral tissues, resulting in an increase in the serum concentration of FT4 following oral propranolol administration. Furthermore, with the increase in the concentration of FT4, the negative feedback from serum FT4 can inhibit the secretion of STSH, leading to a decrease in the sTSH concentration. Despite the observed changes in this study, however, the concentrations of FT4 and sTSH following medication remained within the normal range, and the children did not exhibit any clinical symptoms.

During medication, a few children in the present study exhibited loss of appetite, diarrhea, slow weight gain and certain other phenomena, but these conditions all alleviated spontaneously, without apparent adverse reactions, such as severe hypoglycemia, hypotension or bradycardia. This indicates that propranolol is highly safe; however, the long-term effect of the drug is not clear, and a long-term follow-up is required. In addition, there is no uniform standard regarding when to terminate propranolol administration in the treatment of hemangioma, and there have been numerous reports of propranolol withdrawal leading to the recurrence of the hemangioma (16-19), which necessitated the renewal of the medication administration. An associated study (20) showed that the changes in hemangioma tend to be larger in the first 5 months of treatment and that further treatment effects are less obvious. Zhao et al (10) found that propranolol has no significant effect on involuting hemangiomas; however, the experience of the author led to the recommendation that the administration of propranolol should be slowly stopped following the subsidence of the hemangioma, as this can reduce the vascular tumor recurrence rate. Combining the results of this study and the relevant literature, we suggest that propranolol is a safe and effective option for the treatment of infantile hemangioma and should be considered as a first-line therapy.

Current clinic practice mainly involves the use of oral propranolol tablets for treatment; however, the tablet dose is not easy to control and the tablets are not easy for infants to take. Furthermore, since the gastrointestinal tract absorbs propranolol for liver metabolism and the renal excretion process, the drug exhibits low bioavailability and can easily interact with other medications. In addition, propranolol has a short half-life, and large fluctuations in the blood drug concentration can be observed (21). We therefore suggest that propranolol should be made into an injection or external preparation for local endovascular aneurysm injection or local surface use, which would not only lead to a high local concentration, but also to a reduction in the systemic adverse reactions.

The occurrence and development of numerous types of cancer, in addition to hemangioma, are closely associated with angiogenesis (22). Inflammatory factors and hypoxia can induce the expression of VEGF, and the combination of VEGF and the VEGF receptor $1 / 2 / 3$ can activate the phosphoinositide 3-kinase/Akt, p38MAPK and FAK signaling pathway (22). The use of VEGF and its receptor as a target is an area of particular focus in the development of anticancer drugs, and further studies are required to determine whether propranolol can be developed as an adjuvant cancer treatment. 
In conclusion, oral propranolol has a good curative effect in the treatment of proliferative hemangioma, with few side effects and a high level of safety. The mechanism underlying the effects of propranolol may be associated with the downregulation of VEGF, bFGF and MMP-9 expression.

\section{Acknowledgements`}

This study was supported by the Class B Science and Technology Project of the Education Department of Fujian Province (no. JB11055), the Scientific Research Fund project of the National Health and Family Planning Committee-Fujian Province Joint Research Project of Health Education (no. WKJ-FJ-03) and the National Key Clinical Specialty Discipline Construction Program.

\section{References}

1. Schwartz RA, Sidor MI, Musumeci ML, et al: Infantile hemangiomas: A challenge in pediatric dermatology. J Eur Acad Dermatol Venereol 24: 631-638, 2010.

2. Jinnin M, Ishihara T, Boye E, et al: Recent progress in studies of infantile hemangioma. J Dermatol 37: 283-298, 2010.

3. Boye E and Olsen BR: Signaling mechanisms in infantile hemangioma. Curr Opin Hematol 16: 202, 2009.

4. Zhao ZF, Lü RR, Huo R, Fu HB and Xu GQ: The change of serum vascular endothelial growth factor and matrix metalloproteinases-9 in proliferative hemangioma treated with propranolol. Zhong Hua Zheng Xing Wai Ke Za Zhi 27: 359-361, 2011 (In Chinese).

5. Liu D: Propranolol treatment of infantile proliferation hemangiomas experimental study (unpublished $\mathrm{PhD}$ thesis). Luzhou Medical College, 2012 (In Chinese).

6. Léauté-Labrèze C, Dumas de la Roque E, Hubiche T, et al: Propranolol for severe hemangiomas of infancy. N Engl J Med 358: 2649-2651, 2008.

7. Achauer BM, Chang CJ and Vander Kam VM: Management of hemangioma of infancy: Review of 245 patients. Plast Reconstr Surg 99: 1301-1308, 1997.
8. Hu Q, Lin X, Shang Q, et al: The determination and significance of VEGF in the serum of hemangioma patients. Zhong Hua Zheng Xing Wai Ke Za Zhi 18: 98-100, 2002 (In Chinese).

9. Zhang L, Lin X, Wang W, et al: Clinical implications of serum VEGF concentration in hemangioma. Zhong Hua Xiao Er Wai Ke Za Zhi 25: 11-12, 2004 (In Chinese).

10. Zhao Z, Lv R, Huo R, et al: Change of bFGF in patients with hemangioma treated with oral Propranolol. Zhong Guo Mei Rong Zheng Xing Wai Ke Za Zhi 22: 504-507, 2011 (In Chinese).

11. Folkman J: Clinical application of research on angiogenesis. N Engl J Med 333: 1757-1763, 1995.

12. Fredriksson JM, Lindquist JM, Bronnikov GE and Nedergaard J: Norepinephrine induces vascular endothelial growth factor gene expression in brown adipocytes through a beta-adrenoreceptor/cAMP/protein kinase A pathway involving Src but independently of Erk1/2. J Biol Chem 275: 13802-13811, 2000.

13. Su Y, Huang X, Raskovalova T, et al: Cooperation of adenosine and prostaglandin E2 (PGE2) in amplification of cAMP-PKA signaling and immunosuppression. Cancer Immunol Immunother 57: 1611-1623, 2008.

14. Roesler WJ, Vandenbark GR and Hanson RW: Cyclic AMP and induction of eukaryotic gene transcription. J Biol Chem 263: 9063-9066, 1998.

15. Gelmetti C, Frasin A and Restano L: Innovative therapeutics in pediatric dermatology. Dermatol Clin 28: 619-629, 2010.

16. Sans V, Roque ED, Berge J, et al: Propranolol for severe infantile hemangiomas: Follow-up report. Pediatrics 124: e423-e431, 2009.

17. Schupp CJ,Kleber JB, Günther P and Holland-Cunz S: Propranolol therapy in 55 infants with IH: Dosage, duration, adverse effects and outcome. Pediatr Dermatol 28: 640-644, 2011.

18. Georgountzou A, Karavitakis E, Klimentopoulou A, et al: Propranolol treatment for severe infantile hemangiomas: A single-centre 3-year experience. Acta Paediatr 101: e469-e474, 2012.

19. Chang L, Ma G, Jin Y, et al: Recurrence of infantile hemangioma after termination of propranolol treatment. Ann Plast Surg 72: 173-175, 2014.

20. Bagazgoitia L, Torrelo A, Gutiérrez JC, et al: Propranolol for infantile hemangiomas. Pediatr Dermatol 28: 108-114, 2011.

21. He SY, Zhang J and Ding JS: The application research progress and mechanism of Propranolol treaing on Hemangioma. Zhong Nan Yao Xue 9: 274-276, 2011 (In Chinese).

22. Xiong B and Yi C: Vascular endothelial growth factor family and gene therapy. Zhong Hua Yi Xue Mei Xue Mei Rong Za Zhi 12: 328-330, 2000 (In Chinese). 